\title{
Preoperative Varus-Valgus Stress Angle Difference Is Valuable for Predicting the Extent of Medial Release in Varus Deformity during Total Knee Arthroplasty
}

\author{
O-Sung Lee, $\mathrm{MD}^{1}$, Ashraf Elazab, $\mathrm{MD}^{2,3}$, and Yong Seuk Lee, $\mathrm{MD}^{3}$ \\ ${ }^{1}$ Department of Orthopaedic Surgery, Mediplex Sejong Hospital, Incheon, Korea; ${ }^{2}$ Department of Orthropaedic Surgery, Dammam Medical Complex, Dammam, Saudi \\ Arabia; ${ }^{3}$ Department of Orthopaedic Surgery, Seoul National University Bundang Hospital, Seoul National University College of Medicine, Seongnam, Korea
}

\begin{abstract}
Purpose: The purpose of this study was to compare the predictive value of the valgus stress angle (SA), varus SA and varus-valgus SA difference (VVD) in predicting the degree of medial release in varus deformity during total knee arthroplasty (TKA).

Materials and Methods: One hundred eight TKAs (78 patients), which were performed in primary osteoarthritis with varus knee deformity, were retrospectively classified into three groups according to the degree of medial release (group A, mild release; group B, moderate release; and group $\mathrm{C}$, severe release). Medical charts were reviewed, and long weight bearing standing anteroposterior radiographs and varus-valgus stress radiographs were evaluated.

Results: The valgus SA was significantly different between group A and B and between group A and C. However, it was not significantly different between group B and C. The varus SA was significantly different between group B and C and between group A and C. However, it was not significantly different between group A and B. The VVD was significantly different in all intergroup comparisons.

Conclusions: Evaluation of the VVD is more valuable in predicting the degree of medial release in TKA performed in varus knee deformity than evaluation of the simple valgus and varus SA.
\end{abstract}

Keywords: Knee, Arthroplasty, Varus, Varus-valgus stress angle difference, Medial release

\section{Introduction}

Proper soft tissue balance is essential for a successful total knee arthroplasty (TKA) and incorrect soft tissue balancing can result in catastrophic complications ${ }^{1-5)}$. In particular, varus knees are often followed by progressive contracture of the medial soft tissues and laxity of the lateral soft tissues. Therefore, proper medial re-

Received May 22, 2018; Revised (1st) August 30, 2018;

(2nd) September 30, 2018; (3rd) October 24, 2018;

Accepted November 15, 2018

Correspondence to: Yong Seuk Lee, MD

Department of Orthopaedic Surgery, Seoul National University Bundang

Hospital, Seoul National University College of Medicine, 82 Gumi-ro

173beon-gil, Bundang-gu, Seongnam 13620, Korea

Tel: +82-31-787-7199, Fax: +82-31-787-4056

E-mail: smcos1@hanmail.net

This is an Open Access article distributed under the terms of the Creative Commons Attribution Non-Commercial License (http://creativecommons.org/licenses/by-nc/4.0/) which permits unrestricted non-commercial use, distribution, and reproduction in any medium, provided the original work is properly cited. leases of the medial ligaments are required for successful TKA in order to realign the leg and to achieve balance ${ }^{6-9)}$. Gradual release of subperiosteal elevation of the medial collateral ligament (MCL) followed by posteromedial capsule detachment and semimembranosus release from the proximal tibia has been widely used for the correction of varus deformity. However, the optimal methods of medial release are still controversial because they are highly dependent on the surgeon in most cases ${ }^{10,11)}$.

During the medial release procedure, an excessive medial release could result in gross instability of the tibiofemoral joint, which may indicate the need for a more costly and functionallyrestrictive hinged $\mathrm{TKA}^{2,7,9,12,13)}$. Furthermore, incorrect balancing can result in abnormal and increased loading leading to excessive polyethylene wear, osteolysis, and loosening of the implant ${ }^{6,14)}$. Therefore, accurate preoperative planning is essential.

Currently, the varus and valgus stress views are regarded as a more useful tool than a simple radiograph for the evaluation of medial contracture and lateral laxity. However, the correlation between the simple valgus stress angle (SA) and the extent of 
the medial release has not been determined ${ }^{15,16}$. Identification of relation between the stress radiograph and the extent of medial release would be useful in preoperative planning. However, the simple valgus SA cannot reflect lateral laxity, and the varus SA alone cannot predict the medial contracture of a varus knee. Therefore, we have been using the varus-valgus SA difference (VVD) for predicting the extent of medial release preoperatively under the hypothesis that it would be a more valuable parameter for predicting the extent of medial release compared to the simple varus and valgus SA. The purpose of this study was to compare the usefulness of the simple valgus and varus SA and the VVD in predicting the degree of medial release in TKA.

\section{Materials and Methods}

One hundred forty-seven TKAs, which were performed by a single surgeon from March 2014 to March 2015, were retrospectively reviewed. The inclusion criteria were patients with primary osteoarthritis and preoperative full length standing anteroposterior (AP) radiographs showing varus deformity. Exclusion criteria were as follows: (1) secondary osteoarthritis such as posttraumatic arthritis and sequelae of septic arthritis, (2) inflammatory arthritis, (3) previous history of surgery on the operating knee, and (4) lateral compartment osteoarthritis with valgus deformity. Finally, revision cases (13 knees), cases with a diagnosis other than primary osteoarthritis (5 knees with osteonecrosis), and cases utilizing another TKA implant (21 knees) rather than our most commonly used posterior cruciate substituting implant (Lospa knee system; Corentec Co., Seoul, Korea) were excluded in order to eliminate implant-related selection bias. Thus, our final cohort consisted of 73 female and 5 male patients who underwent 108 TKAs ( 55 left and 53 right knees). There was no case of the constrained condylar knee prosthesis (Fig. 1). The mean age of patients was $72.4 \pm 5.8$ years. This study was approved by the Institutional Review Board of our hospital.

\section{Surgical Technique}

After performing bone cuts perpendicular to the mechanical axis of the femur and mechanical axis of the tibia, osteophytes were removed. Both the anterior cruciate ligament and posterior cruciate ligament were sacrificed. Electrocautery was used to release the soft tissue from the medial edge of the tibial plateau. Subsequently, the tibial size was determined and the remaining portion of the tibial bone was also resected. Then, the medial and lateral gaps were measured in full extension and $90^{\circ}$ of flexion using a tension gauge. First, measurement of the mediolateral (ML) gap was performed using a tension gauge and scaled-force forceps in $90^{\circ}$ knee flexion position. Medial and lateral tensors were distracted independently using scaled-force forceps with manual maximal tension until the medial and lateral ligaments were tight. In all knees, the medial gap was tighter than the lateral gap until a balanced gap was achieved. A medial release was usually performed if the medial and lateral gap difference in flexion or extension was larger than $2 \mathrm{~mm}$. The release was done in a gradual manner and the flexion and extension gaps were rechecked after each degree of release. Finally, the ML gap differences in both flexion and extension were less than $2 \mathrm{~mm}$ in all cases in this study.

\section{Evaluations}

Measurements were made using PACS digital radiographic software (INFINITT ver. 5.0.9.2, Seoul, Korea). Pre- and postoperative long weight bearing standing AP radiographs were used for measurements of the hip-knee-ankle (HKA) angle. Intraoperative data were recorded carefully after each operation including the degree of medial release. Preoperative stress radiographs were taken using a Telos SE arthrometer (Fa Telos, MedizinischTechnische, Greisheim, Germany) with a $15 \mathrm{lb}(6.8 \mathrm{~kg})$ force at $30^{\circ}$ of knee flexion. A line that passes the midpoint of the distal femoral shaft at $10 \mathrm{~cm}$ and $15 \mathrm{~cm}$ from the knee joint line was drawn. Another line that passes the midpoint of the proximal tibial shaft at $7.5 \mathrm{~cm}$ and $12.5 \mathrm{~cm}$ from the knee joint line was drawn. The angle between these two lines was defined as the valgus SA on valgus stress radiographs (Fig. $2 \mathrm{~A})^{17}$. The varus SA was also measured as the angle between these two lines on varus stress radiographs (Fig. 2B). The VVD was defined as the abso-

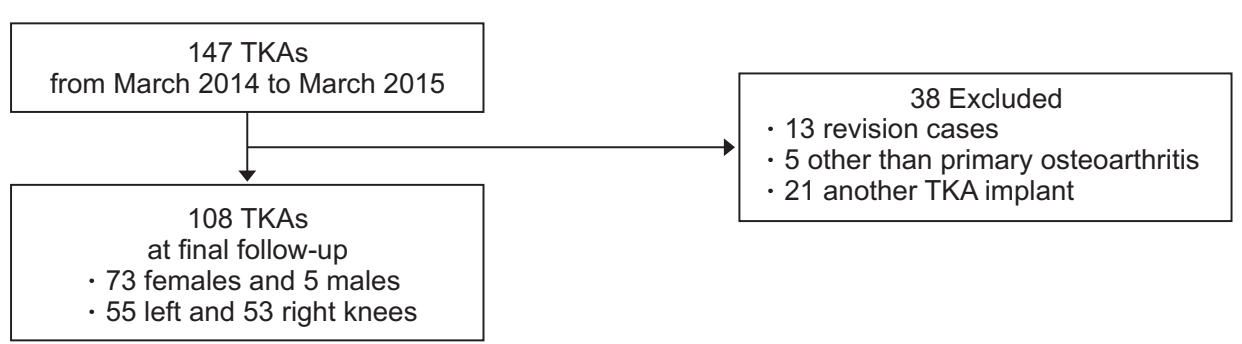

Fig. 1. Patient flow diagram. TKA: total knee arthroplasty. 

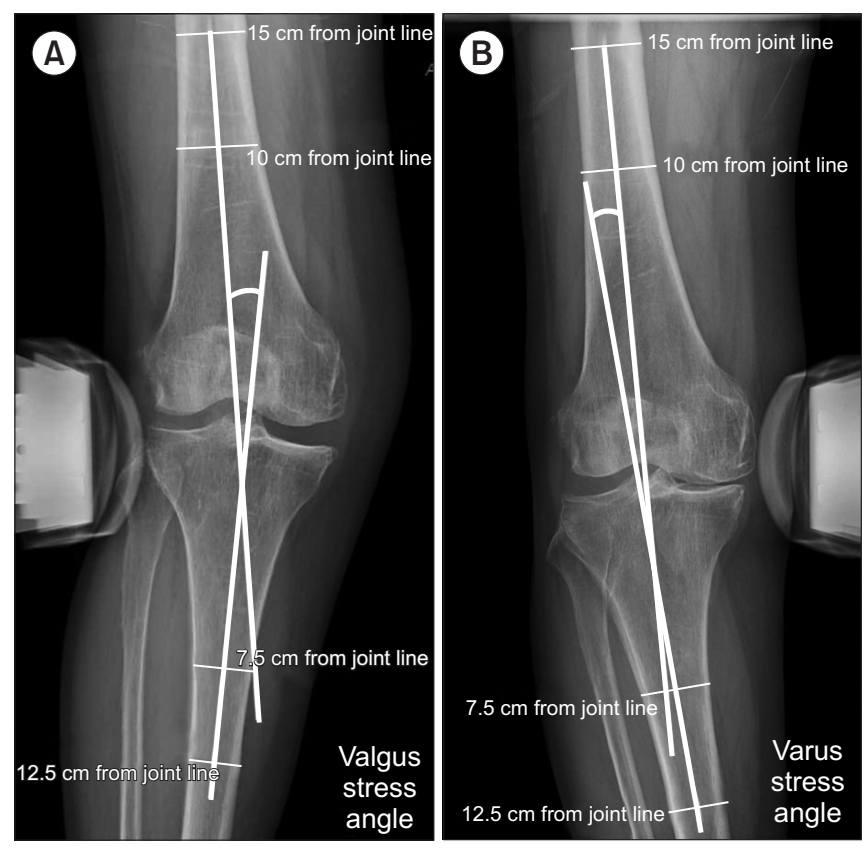

Fig. 2. (A) On the valgus stress radiograph, a line that passes the midpoint of the distal femoral shaft at $10 \mathrm{~cm}$ and $15 \mathrm{~cm}$ from the knee joint line was drawn. Another line that passes the midpoint of the proximal tibial shaft at $7.5 \mathrm{~cm}$ and $12.5 \mathrm{~cm}$ from the knee joint line was drawn. The angle between these two lines was defined as the valgus stress angle. (B) On the varus stress radiograph, a line that passes the midpoint of the distal femoral shaft at $10 \mathrm{~cm}$ and $15 \mathrm{~cm}$ from the knee joint line was drawn. Another line that passes the midpoint of the proximal tibial shaft at $7.5 \mathrm{~cm}$ and $12.5 \mathrm{~cm}$ from the knee joint line was drawn. The angle between these two lines was defined as the varus stress angle. lute difference between the varus and valgus SAs.

Mild release was done for all patients and it was defined as a simple release of the soft tissue from the medial margin of the tibial plateau. Additionally, medial anterior release was done for a tight flexion gap, and medial posterior release was performed for a tight extension gap. A grade I release of medial structure was defined as a half-distance release from the medial joint line to the meta-diaphyseal junction of the proximal tibia. A grade II release of medial structure was defined as a full-distance release from the medial joint line to the meta-diaphyseal junction (Fig. 3). If grade II release was not enough to achieve adequate gap balancing within a $2 \mathrm{~mm}$ difference, the "pie crust" technique was performed with a number of snips at the medial collateral ligament using a 17-gauge spinal needle ${ }^{18,19)}$. We performed the pie crust on the posterior fiber of the MCL in the extension position of knee joint when the joint gap was distracted with a tension gauge and scaled-force forceps. After every 5 to 6 times of snips, the gap was distracted gently with the scaled-force forceps. The procedures and gap checking were repeated until an appropriate ML balance of the extension gap was obtained by manipulation with a repeated valgus test while the tension gauge was in place. The flexion gap was also measured using the tension gauge and scaled-force forceps. If the medial flexion gap was still tight, the pie crust on the anterior fiber of the superficial MCL and gentle spreading with the scaled-force forceps were repeated. If the ML balance could not be achieved after the pie crust, additional medial epicondylectomy was performed.

Finally, patients were divided into three groups according to the
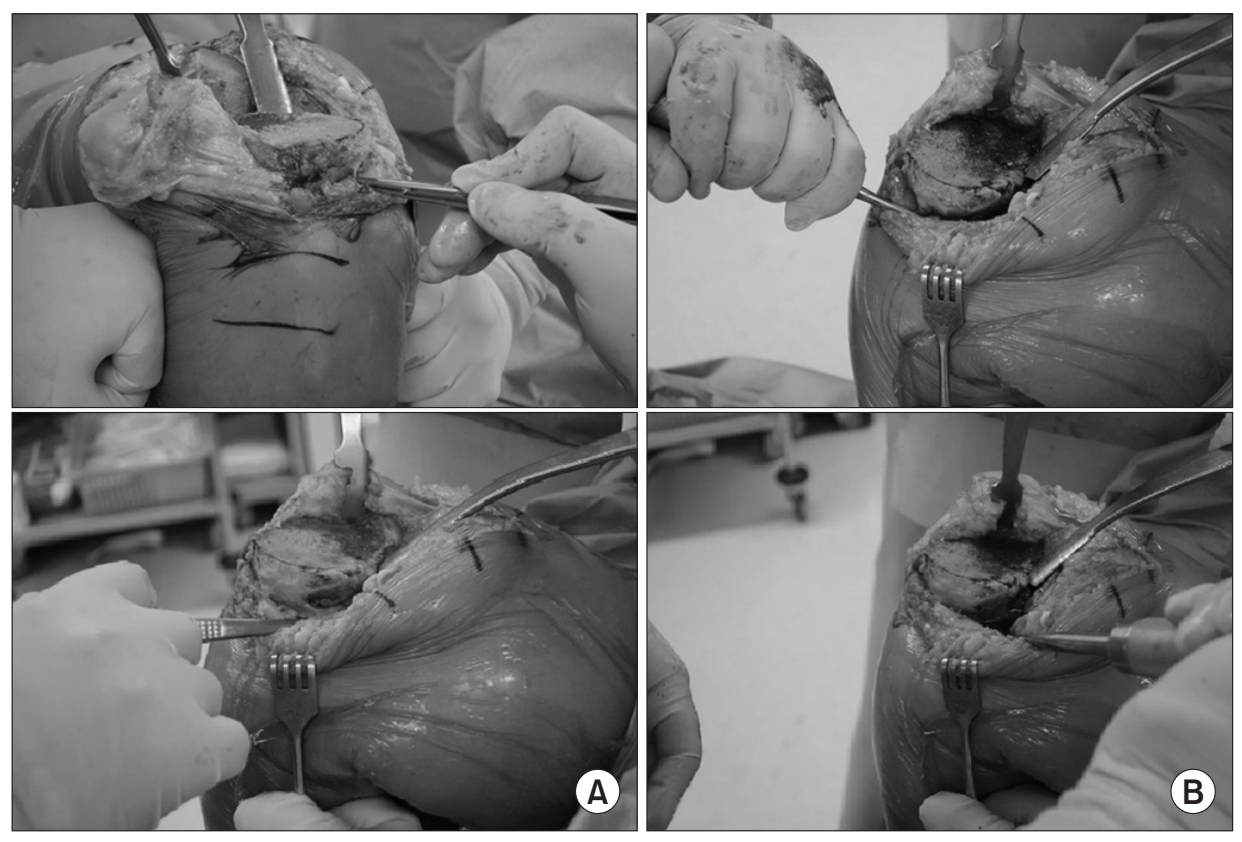

Fig. 3. (A) Intraoperative photographs demonstrating the level of grade I release (up to the midpoint between the joint line and the meta-diaphyseal junction). (B) Intraoperative photographs demonstrating the level of grade II release (to the metadiaphyseal junction). 
degree of medial release to verify our hypothesis: group A, mild release; group B, moderate release; and group $\mathrm{C}$, severe release. Group A had only mild release regardless of the anterior and posterior portions. Group B required grade I or II release on the medial anterior or posterior structures except for the cases which required grade II release on both medial anterior and posterior structures. Group C included cases requiring grade II releases at both the medial anterior and posterior portions, additional pie crust, and medial epicondylectomy (Table 1$)^{18,19)}$.

\section{Statistical Analysis}

All statistical analyses were performed with IBM SPSS ver. 22.0 (IBM Corp., Armonk, NY, USA). Multiple analyses of variance tests were used to determine the difference and correlation between the different measurements of preoperative VSA, VVD, and HKA angle as well as postoperative HKA angle and medial release in the three groups. Groups were compared by Tukey's test. A p-value $<0.05$ was considered to be significant.

\section{Results}

According to the type of medial release, 108 knee cases were categorized into three groups; 45, 42 and 21 knees in groups $\mathrm{A}, \mathrm{B}$, and $\mathrm{C}$, respectively. Mean values of the valgus SA were $6.57^{\circ} \pm 3.98^{\circ},-3.53^{\circ} \pm 6.15^{\circ}$, and $-5.14^{\circ} \pm 6.08^{\circ}$ in groups $\mathrm{A}, \mathrm{B}$, and $\mathrm{C}$, respectively. In a sub-analysis, there were significant differences between group A and B $(\mathrm{p}<0.001)$ and between group A and $C(p<0.001)$. However there was no significant difference between group $B$ and $C(p=0.499)$. In terms of the varus $S A$, mean values of the varus $S A$ were $22.33^{\circ} \pm 4.93^{\circ}, 23.23^{\circ} \pm 5.79^{\circ}$, and $16.61^{\circ} \pm 9.18^{\circ}$ in groups $\mathrm{A}, \mathrm{B}$, and $\mathrm{C}$, respectively. The varus SA was significantly different between group B and $C(p<0.001)$ and between group $A$ and $C(p=0.002)$. However, it was not significantly different between group A and $B(p=0.781)$. VVD measurements were $28.9^{\circ} \pm 6.74^{\circ}, 19.7^{\circ} \pm 8.4^{\circ}$, and $11.5^{\circ} \pm 9.42^{\circ}$ in groups $\mathrm{A}, \mathrm{B}$, and $\mathrm{C}$, respectively. In a sub-analysis, significant differences were noted among all groups (between $\mathrm{A}$ and $\mathrm{B}, \mathrm{A}$ and $\mathrm{C}$, and B and C) (all p<0.001) (Tables 1 and 2).

Preoperative weight bearing HKA angle measurements were $-9.2^{\circ} \pm 5.4^{\circ},-10.86^{\circ} \pm 4.91^{\circ}$, and $-13.55^{\circ} \pm 6.55^{\circ}$ in groups $\mathrm{A}, \mathrm{B}$, and $\mathrm{C}$, respectively (Table 1 ). There was statistical difference in the preoperative HKA angle measurements only between group A and $\mathrm{C}(\mathrm{p}=0.009)$. Postoperative HKA angle measurements were $0.50^{\circ} \pm 2.40^{\circ}, 0.95^{\circ} \pm 2.75^{\circ}$, and $0.45^{\circ} \pm 3.79^{\circ}$ for groups $\mathrm{A}, \mathrm{B}$, and $\mathrm{C}$, respectively. There was no statistical difference in the postoperative HKA angle measurements among the three groups (Table 2).

Table 2. Intergroup Difference for Each Parameter

\begin{tabular}{lllrr}
\hline \multicolumn{1}{c}{ Parameter } & \multicolumn{2}{c}{ Group } & Mean difference & p-value \\
\hline Preop HKA & A & B & $1.66 \pm 1.17$ & 0.336 \\
angle $\left(^{\circ}\right)$ & B & C & $2.70 \pm 1.46$ & 0.160 \\
& C & A & $-4.36 \pm 1.44$ & 0.009 \\
Preop valgus & A & B & $10.10 \pm 1.14$ & $<0.001$ \\
SA $\left(^{\circ}\right)$ & B & C & $1.61 \pm 1.43$ & 0.499 \\
& C & A & $-11.7 \pm 1.41$ & $<0.001$ \\
Preop varus & A & B & $0.90 \pm 1.24$ & 0.781 \\
SA $\left(^{\circ}\right)$ & B & C & $-6.62 \pm 2.21$ & $<0.001$ \\
& C & A & $5.72 \pm 2.42$ & 0.002 \\
Preop varus-valgus & A & B & $9.20 \pm 1.71$ & $<0.001$ \\
SA difference $\left(^{\circ}\right)$ & B & C & $8.23 \pm 2.13$ & $<0.001$ \\
& C & A & $-17.43 \pm 2.10$ & $<0.001$ \\
Postop HKA & A & B & $-0.45 \pm 0.61$ & 0.741 \\
angle $\left({ }^{\circ}\right)$ & B & C & $0.51 \pm 0.76$ & 0.784 \\
& C & A & $-0.06 \pm 0.75$ & 0.997 \\
\hline
\end{tabular}

Values are presented as mean \pm standard deviation.

Preop: preoperative, HKA: hip-knee-ankle, (-): values for varus angles, SA: stress angle, Postop: postoperative.

Table 1. Comparison of Different Angles in Three Groups

\begin{tabular}{lccc}
\hline \multicolumn{1}{c}{ Parameter } & Group A (mild release) & Group B (moderate release) & Group C (severe release) \\
\hline No. & 45 & 42 & 21 \\
Varus SA $\left(^{\circ}\right)$ & $-22.33 \pm 4.93$ & $-23.23 \pm 5.79$ & $-16.61 \pm 9.18$ \\
Valgus SA $\left(^{\circ}\right)$ & $6.57 \pm 3.98$ & $-3.53 \pm 6.15$ & $-5.14 \pm 6.08$ \\
Varus-valgus SA difference $\left(^{\circ}\right)$ & $28.9 \pm 6.74$ & $19.7 \pm 8.4$ & $11.5 \pm 9.42$ \\
Preoperative HKA angle $\left(^{\circ}\right)$ & $-9.2 \pm 5.4$ & $-10.86 \pm 4.91$ & $-13.55 \pm 6.55$ \\
Postoperative HKA angle $\left(^{\circ}\right)$ & $0.50 \pm 2.40$ & $0.95 \pm 2.75$ & $0.45 \pm 3.79$ \\
Postoperative WBR & $53 \pm 9$ & $54 \pm 11$ & $52 \pm 14$ \\
\hline
\end{tabular}

Values are presented as mean \pm standard deviation.

SA: stress angle, (-): values for varus angles, HKA: hip-knee-ankle, WBR: weight bearing ratio. 


\section{Lee et al. Varus-Valgus Stress Angle Diffenrence to Predict Medial Release in TKA}

\section{Discussion}

The principle findings of this study were that 1 ) the varus SA and valgus SA were somewhat correlated with the degree of medial release in the knee and 2) the VVD was more predictive in the severely contracted knee than the simple varus and valgus SA. The valgus SA was significantly different between mild and moderate release groups and between mild and severe release groups. However, it was not significantly different between moderate and severe release groups. The varus SA was significantly different between moderate and severe release groups and between mild and severe release groups. However, it was not significantly different between mild and moderate release groups. The VVD was significantly different in all intergroup comparisons.

Through meticulous preoperative radiographic evaluation, the site and degree of medial release can be predicted ${ }^{7,920)}$. Many attempts have been made to achieve this goal. Some authors suggested that distractive stress radiographs may be more useful than long weight bearing standing AP radiographs for assessing soft tissue balance ${ }^{21}$. In another study, the extent of medial release was correlated with the degree of varus deformity on the preoperative distractive stress radiographs; however, they were not able to predict the extent of the medial release in some patients ${ }^{22}$. Mihalko and $\mathrm{Krackow}^{20)}$ suggested that a preoperative distraction test and a varus-valgus stress test can provide useful information on medial and lateral ligament laxity. However, the relationship between the extent of release and medial and lateral gaps has not been clearly established. Others recommended the valgus SA as a determinant of the degree of medial release ${ }^{15,16)}$. However, some cases with small preoperative valgus SA measurements suspected as poor varus deformity reducibility were corrected without excessive medial release ${ }^{8)}$.

In all patients enrolled in our study, the medial release was made cautiously and gradually. Posterior release for a tight extension gap and anterior release for a tight flexion gap were parts of the initial release. Sequential releases were performed with a grade I/grade II release, pie crust, and medial epicondylectomy. We hypothesized that the VVD may be more useful than the simple valgus SA for predicting the extent of the sequential release. Even though the valgus SA was significantly different between mild and moderate release groups and between mild and severe release groups, it was not useful in comparing moderate and severe groups. Therefore, it can be implied that the valgus SA is a weak predictor of the degree of medial release.

In the cases with severe fixed varus deformity requiring extensive medial release, a constrained condylar implant may be necessary because collateral ligaments cannot be balanced with soft tissue releases, or the MCL can be incompetent or transected. Therefore, accurate preoperative planning is essential. In the present study, the VVD was more predictive for the extent of medial release in all cases even when an extensive release was required. It is probably because the valgus SA did not reflect the lateral laxity which was likely to be accompanied by varus knees. In many cases of long-standing varus deformity, the lateral ligament is deformed and lax. The medial gap should be released to the lax lateral gap in order to achieve a well-balanced gap during a TKA. However, the valgus SA can only evaluate the medial contracture, and it cannot reflect the degree of the lateral laxity. Regarding the clinical relevance of the varus SA, it could be suggested as a parameter to evaluate preoperative lateral laxity. If the preoperative varus SA is large, an extensive medial release can be necessary to balance the lax lateral structures. It requires careful attention to prevent failure of the conventional soft tissue release technique during TKA. However, in the present study, the varus SA did not show a significant difference in all intergroup comparisons.

The VVD also has limitations given the fixed values to be established a firm treatment algorithm: there was an overlap between the groups although the mean VVD was significantly different among all three groups. Therefore, surgeons would need to continue to perform release as described until the knee is balanced. The simple varus \& valgus SA and VVD could be used as adjunctive factors in association with intraoperative findings for proper decision-making to achieve the targeted soft tissue balance. However, the VVD demonstrated a larger difference between groups than the varus and valgus SA. Therefore, the VVD may provide a clearer prediction. In group $\mathrm{A}$, both mean values of varus and valgus $\mathrm{SA}$ were high, which denotes that there was no fixed medial and lateral contracture. In group B, the varus SA was high and the valgus SA was low, which denotes that there was preoperative medial contracture. In group $\mathrm{C}$, both mean values of varus and valgus SA were low, which denotes that there was preoperative medial and lateral contracture. Additionally, regarding the interpretation of the results of the varus SA, we thought that the fact that varus SA (not the varus deformity) was larger in group A than in group $\mathrm{C}$ does not necessarily mean that more release is required in group A. This was because the varus SA, valgus SA, and VVD could be affected by osteophytes and bony deformity as well as medial and lateral laxity of soft tissue. Moreover, the ML balance could be affected by various factors such as the resection amount of the distal femur and proximal tibia, thickness of polyethylene, and sizing of implants as well as the status of the soft tissue. If the ML balance could be achieved by only medial 
release, the varus SA was more predictable than the VVD. However, in this study, we demonstrated that the degree of medical laxity (varus SA) simply does not determine the degree of medial release, which is affected by various preoperative factors, and that VVDs are more predictable. From this study, it can be inferred that preoperative stress views and VVD may be a valuable guideline for assessing the extent of medial release to be performed regardless of the severity of deformity.

There are some limitations of this study, and our findings should be interpreted in light of these. First, the retrospective nature of this study introduces the influence of potential confounders. Second, there are potentially other factors related to the degree of medial release, such as the preoperative degree of flexion contracture and duration of fixed varus deformity. In this study, there was no correlation between the preoperative HKA angle and the degree of medial release. It may be due to lack of evaluation of the contracture of the medial structure caused by preoperative flexion contracture and duration of fixed varus deformity. However, flexion contracture is related to sagittal deformity, not coronal deformity. Therefore, the conclusion of this study is fairly reasonable without the analysis of flexion contracture. Additionally, the duration of varus deformity was not considered in the analysis because it was impossible to objectively investigate upon the patients' memory. Third, all evaluations were performed using only radiological parameters as endpoints, which surgeons use as a proxy for better outcomes; however, clinical results and patients' satisfaction may be different. Moreover, there is a paucity of firmly established methods of measuring stress radiographs. Therefore, there is a possibility of different interpretations with other methods. Additionally, this study lacks statistical power because there are not many cases that require severe release using our gradual release method. Lastly, we did not take into account the economic implications of this study and there would be extra cost, as well as radiation exposure, for stress radiography.

\section{Conclusions}

Evaluation of the VVD is more valuable in predicting the degree of medial release of TKA for varus knee deformity than evaluation of the simple valgus and varus $\mathrm{SA}$.

\section{Conflict of Interest}

No potential conflict of interest relevant to this article was reported.

\section{References}

1. Aunan E, Kibsgard T, Clarke-Jenssen J, Röhrl SM. A new method to measure ligament balancing in total knee arthroplasty: laxity measurements in 100 knees. Arch Orthop Trauma Surg. 2012;132:1173-81.

2. Babazadeh S, Stoney JD, Lim K, Choong PF. The relevance of ligament balancing in total knee arthroplasty: how important is it? A systematic review of the literature. Orthop Rev (Pavia). 2009;1:e26.

3. Incavo SJ, Schmid S, Sreenivas K, Ismaily S, Noble PC. Total knee arthroplasty using anatomic alignment can produce mid-flexion laxity. Clin Biomech (Bristol, Avon). 2013;28: 429-35.

4. King JJ 3rd, Chakravarty R, Cerynik DL, Black A, Johanson NA. Decreased ratios of lateral to medial patellofemoral forces and pressures after lateral retinacular release and gender knees in total knee arthroplasty. Knee Surg Sports Traumatol Arthrosc. 2013;21:2770-8.

5. Walker PS, Heller Y, Cleary DJ, Yildirim G. Preclinical evaluation method for total knees designed to restore normal knee mechanics. J Arthroplasty. 2011;26:152-60.

6. Chen W, Nagamine R, Kondo K, Todo M. Effect of medial soft-tissue releases during posterior-stabilised total knee arthroplasty. J Orthop Surg (Hong Kong). 2011;19:230-3.

7. Chon JG, Sun DH, Jung JY, Kim TI, Jang SW. Rotational alignment of femoral component for minimal medial collateral ligament release in total knee arthroplasty. Knee Surg Relat Res. 2011;23:153-8.

8. Moon YW, Kim JG, Han JH, Do KH, Seo JG, Lim HC. Factors correlated with the reducibility of varus deformity in knee osteoarthritis: an analysis using navigation guided TKA. Clin Orthop Surg. 2013;5:36-43.

9. Mullaji AB, Padmanabhan V, Jindal G. Total knee arthroplasty for profound varus deformity: technique and radiological results in 173 knees with varus of more than 20 degrees. J Arthroplasty. 2005;20:550-61.

10. Hunt NC, Ghosh KM, Athwal KK, Longstaff LM, Amis AA, Deehan DJ. Lack of evidence to support present medial release methods in total knee arthroplasty. Knee Surg Sports Traumatol Arthrosc. 2014;22:3100-12.

11. Sim JA, Lee YS, Kwak JH, Yang SH, Kim KH, Lee BK. Comparison of complete distal release of the medial collateral ligament and medial epicondylar osteotomy during ligament balancing in varus knee total knee arthroplasty. Clin Orthop Surg. 2013;5:287-91. 


\section{Lee et al. Varus-Valgus Stress Angle Diffenrence to Predict Medial Release in TKA}

12. Athwal KK, Hunt NC, Davies AJ, Deehan DJ, Amis AA. Clinical biomechanics of instability related to total knee arthroplasty. Clin Biomech (Bristol, Avon). 2014;29:119-28.

13. Bottros J, Gad B, Krebs V, Barsoum WK. Gap balancing in total knee arthroplasty. J Arthroplasty. 2006;21(4 Suppl 1):11-5.

14. Romero J, Stähelin T, Binkert C, Pfirrmann C, Hodler J, Kessler $\mathrm{O}$. The clinical consequences of flexion gap asymmetry in total knee arthroplasty. J Arthroplasty. 2007;22:235-40.

15. Moon YW, Kim JG, Woo KJ, Lim SJ, Seo JG. Analysis of medial flexion gap after medial release for varus deformity by navigation-guided TKA. Orthopedics. 2011;34:355.

16. Saragaglia D, Chaussard C, Rubens-Duval B. Navigation as a predictor of soft tissue release during 90 cases of computerassisted total knee arthroplasty. Orthopedics. 2006;29(10 Suppl):S137-8.

17. Yagishita K, Muneta T, Ikeda H. Step-by-step measurements of soft tissue balancing during total knee arthroplasty for patients with varus knees. J Arthroplasty. 2003;18:313-20.
18. Kim MW, Koh IJ, Kim JH, Jung JJ, In Y. Efficacy and safety of a novel three-step medial release technique in varus total knee arthroplasty. J Arthroplasty. 2015;30:1542-7.

19. Kwak DS, In Y, Kim TK, Cho HS, Koh IJ. The pie-crusting technique using a blade knife for medial collateral ligament release is unreliable in varus total knee arthroplasty. Knee Surg Sports Traumatol Arthrosc. 2016;24:188-94.

20. Mihalko WM, Krackow KA. Flexion and extension gap balancing in revision total knee arthroplasty. Clin Orthop Relat Res. 2006;446:121-6.

21. Nam SW, Kwak JH, Kim NK, Wang IW, Lee BK. Relationship between tibial bone defect and extent of medial release in total knee arthroplasty. Knee Surg Relat Res. 2012;24:14650 .

22. Sim JA, Kwak JH, Yang SH, Moon SH, Lee BK, Kim JY. Utility of preoperative distractive stress radiograph for beginners to extent of medial release in total knee arthroplasty. Clin Orthop Surg. 2009;1:110-3. 